\title{
SIMILARITY OF STRUCTURES BASED ON MATRIX SIMILARITY
}

\author{
Ivica Kožar, Tea Rukavina, Neira Torić Malić
}

Original scientific paper

The paper presents a numerical procedure for relating the behaviour of two different structures, i.e. determining a scale between two structures. This novel solution is based on the notion of matrix similarity and linear transformations, with the restriction that the scale between structures is determined only after structural discretization, and that both structures have to be in the elastic regime. The structure scale can be determined in loading space or displacement space (i.e. structure forces or displacements are put into relation) where the scaling of the static structure model is based on the matrix equivalence principle, and scaling of the dynamic structure model is based on the Smith normal form. The structure scale in operator space (structure stiffness or flexibility matrices are put into relation) should be based on the Sylvester matrix equation. However, that approach is not practical and is replaced with the Levenberg-Marquardt method for obtaining only approximately equivalent stiffness matrices. Numerical examples illustrate the proposed novel approach.

Keywords: Levenberg-Marquardt method; matrix equivalence; similarity of structures; Smith normal form; structure scales; Sylvester equation

\section{Sličnost konstrukcija zasnovana na sličnosti matrica}

Izvorni znanstveni članak Rad prikazuje numerički postupak za uspostavljanje odnosa između ponašanja dvije različite konstrukcije, odnosno određivanje mjerila (faktora skaliranja) između dvije konstrukcije. Ovo novo rješenje zasnovano je na ideji sličnosti matrica i linearnim transformacijama, uz ograničenja da se mjerilo između konstrukcija određuje tek nakon provođenja diskretizacije te da obje konstrukcije moraju biti u elastičnom području. Mjerilo konstrukcije može se odrediti u polju opterećenja ili pomaka (ovisno o tome dovode li se u vezu sile ili pomaci konstrukcije) gdje se skaliranje statičkog modela konstrukcije zasniva na principu ekvivalentnosti matrica, dok je skaliranje dinamičkog modela konstrukcije bazirano na Smith normalnoj formi. Skaliranje konstrukcije u operatorskom prostoru (matrice krutosti ili fleksije stavljaju se u međuodnos) trebalo bi biti bazirano na Sylvester matričnoj jednadžbi. Međutim, takav pristup nije praktičan te je zamijenjen Levenberg-Marquardt metodom za dobivanje približno ekvivalentnih matrica krutosti. Numerički primjeri ilustriraju predloženi drugačiji pristup.

Ključne riječi: ekvivalentnost matrica; faktori skaliranja konstrukcije; Levenberg-Marquardt metoda; sličnost konstrukcija; Smith normalna forma; Sylvester jednadžba

\section{Introduction}

In structural engineering, there is constantly present an idea of relating two different structures; from the behaviour of one structure, we would like to find out about the behaviour of the other. This is especially pronounced in the case of large structures that are important and usually require monitoring of relevant parameters. Quite often, a model is built hoping that measurements on it would provide us with a better insight into the behaviour of the corresponding large structure. This opens the problem of measurement and parameter transfer between two structures. Data and parameters could be transferred only if we could somehow scale/relate the two structures. This case of scaling of measurements and parameters is described in [1]. Here, we discuss the problem of comparison or scaling of forces and displacements acting on different structures. Also, we would like to scale two structures in the operator space, i.e. put the stiffness or flexibility matrices of different structures into some relation. To a certain level, it can be regarded as making two structures behave equivalently, i.e. having the same displacements under prescribed loading.

An overview of what is usually understood by "scaling" of physical objects is presented in [2]. An attempt to relate structures of different size can be seen in [3], which is based on the Buckingham $\pi$ Theorem [4]. Both approaches are based on dimensional analysis, i.e. structures have to be of the same type and have the same mathematical model that differs only in the value of some scalar parameter. The novel approach presented in the paper is based on the notion of matrix similarity and linear transformations $[5,6]$. The similarity matrix approach should not be confused with the calculation of relevant forces for a given displacement. The similarity matrix $\boldsymbol{S}$ relates all the forces and displacements of two structures; matrix $\boldsymbol{S}$ is independent of any loading and/or displacement values. "Scaling" in this paper means "similarity" and "relating forces and displacements of structures of different size" and is not based on dimensional analysis.

The problem of similarity of structures (or "scaling") is addressed on the general level and is defined for any stable structure, but only after the structure has been discretized. At the moment, the procedure is limited to scaling of elastic structures. Two different structures are related/scaled through use of the scaling matrix $\boldsymbol{I}_{F}$ that relates/puts to scale loading on the structure, and the scaling matrix $\boldsymbol{I}_{S}$ that relates/puts to scale the corresponding displacements. The similarity matrix $\boldsymbol{S}$ is determined through the procedure described in the sequel and the scaling matrices $\boldsymbol{I}_{F}$ and $\boldsymbol{I}_{S}$ are chosen at will. Theoretically, it can take any value or it can be equal to the identity matrix $\boldsymbol{I}$ (in the case when loading and displacements are the same for two structures whose parameters are to be scaled), but in practice it has to be chosen so that the related structure remains in the elastic regime. E.g. if the related structure is 100 times smaller, then the related displacements should be about 100 times smaller, too. When the similarity matrix $\boldsymbol{S}$ is calculated it can be applied to any loading, and the displacements of two structures will always be related by the matrix $\boldsymbol{I}_{S}$ (the same applies to strains and stresses). The similarity matrix $\boldsymbol{S}$ applied to displacements coming from arbitrarily scaled loading of two structures would relate the two 
displacements. In this case, we know the relation between displacements of two different structures under completely different loading.

Static and dynamic loadings have to be treated differently. With static loading, the similarity matrix relates forces and displacements of two structures. Under dynamic loading, an additional similarity matrix scales the mass matrix; the related parameters are eigenvalues and eigenvectors. Scaling of loads and displacements takes place in the vector space; similarity in the operator space relates matrices, which is fundamentally more difficult and requires a different approach.

In the sequel, there is a description of the relating/scaling of structures in the loading space, the displacement space and the operator space and their starting mathematical equations. Part 3 develops the similarity matrix for static and dynamic loading for the related structures. There is the determination of similarity in the operator space based on Levenberg-Marquardt method. Finally, Part 4 illustrates the performance of the procedures with examples of various types of structure similarity calculation.

\section{Similarity of structures \\ 2.1 Similarity in load-displacement space}

Two structures are scaled in order to relate their parameters. To relate two structures that are already discretized they have to be in the same basis, i.e. one of them has to be transformed/scaled to match the basis of the other. Not all parameters of the structure can be easily related at the same time. Assuming that the structure is described with matrix equations, we could relate force vectors $\boldsymbol{F}$ as $\boldsymbol{F}_{1}=\boldsymbol{S}_{F} \boldsymbol{F}_{2}$, displacement vectors $\boldsymbol{d}$ as $\boldsymbol{d}_{1}=\boldsymbol{S}_{d}$ $\boldsymbol{d}_{2}$ where $\boldsymbol{S}$ is some scaling matrix and indices '1' and '2' represent two structures (or the structure and its model). In this paper, displacements $\boldsymbol{d}$ are related in displacement space, forces $\boldsymbol{F}$ are related in load space, and both are vector spaces. The application of similarity/scaling in relating the structure and its model is described in [1].

The novel method described in the paper is general and applicable to all structures where a linear matrix relation is to be established. In addition, a scalar can be introduced anywhere in the relations so that values do not have to be equal but a scalar multiple of each other.

In the basis of the similarity analysis of structures is the matrix equivalence principle [5] $\boldsymbol{B}=\boldsymbol{Q}^{-1} \boldsymbol{A P}$ where matrices $\boldsymbol{A}$ and $\boldsymbol{B}$ are equivalent. In the special case when $\boldsymbol{A}$ and $\boldsymbol{B}$ are of the same dimension and $\boldsymbol{Q}=\boldsymbol{P}$, matrices are similar. Equivalent matrices represent the same linear transformation under two different bases $\boldsymbol{V}$ and $\boldsymbol{W}$. Here we are replacing $\boldsymbol{Q}$ and $\boldsymbol{P}$ with scaling matrices $\boldsymbol{S}_{F}$ and $\boldsymbol{S}_{d}$, to be determined in such a way that matrices $\boldsymbol{A}$ and $\boldsymbol{B}$ are equivalent (for similarity in load-displacement space) or similar (for similarity in operator space). Matrices $\boldsymbol{A}$ and $\boldsymbol{B}$ correspond to the structure stiffness matrices $\boldsymbol{K}_{\text {structure }}$ and $\boldsymbol{K}_{\text {model }}$ or $\boldsymbol{K}_{1}$ and $\boldsymbol{K}_{2}$. If structure stiffness matrices $\boldsymbol{K}$ have different rank, i.e. if we want to relate models with different degrees of freedom, a special procedure for determining the scaling matrix $\boldsymbol{S}$ is used. In that case we cannot say that global (uncondensed) stiffness matrices $\boldsymbol{K}_{\text {structure }}$ and $\boldsymbol{K}_{\text {model }}$ are now similar/equivalent (two matrices are similar/equivalent if and only if they have the same rank, so only condensed matrices can be considered similar/equivalent). However, as it will be seen from the examples, all the required properties are retained and the two structures have the desired properties (e.g. equal displacements for any type of loading).

Note: Similarity matrix $S$ should not be confused with calculation of relevant forces for given displacements, i.e. we have displacements $\boldsymbol{d}_{1}$ from the structure modelled with $\boldsymbol{K}_{1}$ and we are seeking the force vector $\boldsymbol{F}_{2}$ that produces the same displacements on the structure modelled with $\boldsymbol{K}_{2}$. The similarity matrix $S$ relates all the forces and displacements in two structures modelled with stiffness matrices $\boldsymbol{K}_{2}$ and $\boldsymbol{K}_{1}$, i.e. vector $\boldsymbol{F}_{1}=\boldsymbol{S}_{F} \boldsymbol{F}_{2}$ will always produce displacements $\boldsymbol{d}_{1}=\boldsymbol{S}_{d} \boldsymbol{d}_{2}$, there is no need for any recalculation once we have obtained $\boldsymbol{S}$. In other words, $\boldsymbol{S}$ puts forces $\boldsymbol{F}_{2}$ and $\boldsymbol{F}_{1}$ into such a relation that the constraint $\boldsymbol{d}_{1}=\boldsymbol{S}_{S} \boldsymbol{d}_{2}$ is always satisfied.

\subsection{Similarity in operator space}

In this work, operators are stiffness matrices $\boldsymbol{K}_{1}$ and $\boldsymbol{K}_{2}$. Operators could be flexibility matrices as well, since with flexibility matrices in the place of stiffness matrices only $\boldsymbol{F}$ and $\boldsymbol{d}$ exchange their places.

In linear algebra, two matrices $\boldsymbol{A}$ and $\boldsymbol{B}$ are similar if $\boldsymbol{B}=\boldsymbol{P}^{-1} \boldsymbol{A P}$ where $\boldsymbol{A}$ and $\boldsymbol{B}$ are both $[n \times n]$. Similarity is equivalence relation on the space of square matrices. It can be determined from Jordan form of a matrix or from Smith normal form.

This case of similarity is obtained if we apply only one similarity matrix for both displacements and forces so the matrix structural equation becomes $\boldsymbol{K}_{2} \boldsymbol{d}_{2}=\boldsymbol{S}_{S} \boldsymbol{F}_{1}$ or $\boldsymbol{K}_{2} \boldsymbol{S}_{S} \boldsymbol{d}_{1}=\boldsymbol{S}_{S} \boldsymbol{F}_{1}$, which results in the homogeneous Sylvester equation $\boldsymbol{K}_{2} \boldsymbol{S}_{S}-\boldsymbol{S}_{S} \boldsymbol{K}_{1}=\boldsymbol{0}$. Here, for the sake of simplicity, we have assumed that structures have been scaled in spaces of the same size, i.e. structural matrices have been statically condensed $\boldsymbol{K}_{c o n d}=\boldsymbol{K}_{p p}-\boldsymbol{K}_{p s} \boldsymbol{K}_{s s}{ }^{-1} \boldsymbol{K}_{s p}$, where $\boldsymbol{K}_{s S}$ is the matrix part to be condensed and $\boldsymbol{K}_{p p}$ is the part to be kept. However, there is no need for static condensation, as it will be demonstrated in the sequel.

Sylvester matrix equation is of the form

$A X+X B=C$

where $\boldsymbol{X}, \boldsymbol{C} \in \mathfrak{R}^{m \times n}$ and $\boldsymbol{A} \in \mathfrak{R}^{m \times m}, \boldsymbol{B} \in \mathfrak{R}^{n \times n}$. The matrix

$\boldsymbol{X}$ is here named the scaling matrix $\boldsymbol{S}$ and matrices $\boldsymbol{A}$ and $\boldsymbol{B}$ correspond to the structure stiffness matrices $\boldsymbol{K}_{\text {structure }}$ and $\boldsymbol{K}_{\text {model }}$ or $\boldsymbol{K}_{1}$ and $\boldsymbol{K}_{2}$. In the analysis of similarity of structures, we are determining the scaling matrix $\boldsymbol{S}$, in such a way that matrices $\boldsymbol{A}$ and $\boldsymbol{B}$ become similar.

The equation belongs to the class of Lyapunov equations used in system analysis (Lyapunov equation is a special case when $\boldsymbol{B}=\boldsymbol{A}^{\mathrm{T}}$ ) see [6]. Note that $\boldsymbol{A}$ and $\boldsymbol{B}$ do not have to be (asymptotically) stable matrices in order that solution of Eq. (1) exists. The only requirement for $\boldsymbol{X}$ to exist is that matrices $\boldsymbol{A}$ and $-\boldsymbol{B}$ have no eigenvalues in common.

In this work, a homogeneous type of the Sylvester equation is used, i.e. $\boldsymbol{C}=0$. In this case, a nontrivial solution for matrix $\boldsymbol{X}$ exists if and only if matrices $\boldsymbol{A}$ and 
$\boldsymbol{B}$ have some eigenvalues in common. This is easily seen if Eq. (1) is written in the Kronecker product notation

$$
\left(\boldsymbol{I}_{n} \otimes \boldsymbol{A}+\boldsymbol{B} \otimes \boldsymbol{I}_{n}\right) \operatorname{vec} \boldsymbol{X}=\operatorname{vec} \boldsymbol{C}
$$

where $v e c \boldsymbol{X}$ stands for the matrix $\boldsymbol{X}$ written in column form and $\operatorname{vec} \boldsymbol{C}=0$ for the homogeneous type of equation. In the case of the homogeneous Eq. (1) and matrices $\boldsymbol{A}$ and $\boldsymbol{B}$ being of the same rank, we obtain the similarity equation, e.g. see [5]. Matrices $\boldsymbol{A}$ and $\boldsymbol{B}$ are now similar and share many properties, among others they have the same eigenvalues. Matrix $\boldsymbol{X}$ changes the basis of matrix $\boldsymbol{A}$ to the basis of matrix $\boldsymbol{B}$, i.e. similar matrices represent the same linear transformation under different basis.

A closed form solution to the Sylvester matrix equation has been found only recently and it is in the form of a polynomial of known matrices [7]. This type of solution is not practical for our purpose and is replaced with Levenberg-Marquardt procedure for determination of relevant structure parameters [8].

\subsection{Levenberg-Marquardt procedure}

The problem of matrix similarity has been reformulated into the problem of simultaneous determination of structural parameters so that two structure stiffness matrices become similar. The criterion taken for parameter determination is the required equivalence of the displacement vector. The problem belongs to a class of optimization problems that can be written as a minimization of the L2 norm

$$
\boldsymbol{e}=\sum_{i=0}^{n}\left(\delta_{i}^{m}-\delta_{i}\right)^{2}
$$

Parameters $\delta$ in the example are stiffness properties of various structural elements and we have applied Levenberg-Marquardt method [9] for solving Eq. (3). Stiffness parameters cannot be zero, so the method has to be adapted to form an iterative procedure

$$
\boldsymbol{w}_{k+1}=\boldsymbol{w}_{k}-\left(\boldsymbol{J}^{T} \boldsymbol{J}\right)^{-1} \times \boldsymbol{J}^{T} * \boldsymbol{e}
$$

Unfortunately, this procedure is non-linear and the result obtained is valid for only one loading case. The optimal solution is obtained as an intersection of solutions of all relevant load cases.

\section{Formulation of the similarity matrix 3.1 Similarity of structures under static loading}

In the case of static loading, the structural model is described with the well-known matrix equation $\boldsymbol{K} \boldsymbol{d}=\boldsymbol{F}$ where $\boldsymbol{K}$ is the stiffness matrix, $\boldsymbol{d}$ is the displacement vector and $\boldsymbol{F}$ is the loading vector. We are relating a model to the structure so that loading forces and displacements of both structures are simply scaled, i.e. scaled with a constant so that, after scaling we obtain $\boldsymbol{d}_{s t r c t}=\boldsymbol{I}_{S} \boldsymbol{d}_{m d l} ; \boldsymbol{I}_{S}=\left[\begin{array}{ccc}I_{1} & 0 & 0 \\ 0 & \ldots & 0 \\ 0 & 0 & I_{n}\end{array}\right]$

where $\boldsymbol{d}$ is the displacement vector of related nodes ('strct' $=$ structure, ' $\mathrm{mdl}$ ' $=$ model), and $\boldsymbol{I}_{S}$ is the diagonal scaling matrix (simply invertible).

Theoretically, the diagonal scaling matrix $\boldsymbol{I}_{S}$ can take any value but in practice displacements have to be scaled so that the structure (the model) remains in the elastic regime. In the sequel, we will assume $\boldsymbol{I}_{S}=\boldsymbol{I}$ for the sake of simplicity (for dynamic loading the scaling matrix $\boldsymbol{I}_{S}$ will enter the equations). Also, index ' 1 ' will indicate the structure (instead of 'strct') and index ' 2 ' the model (instead of 'mdl'). We will assume that there is a (static) similarity matrix $\boldsymbol{S}_{S}$ that scales loading forces in such a way that the displacement vector constraint from Eq. (5) is satisfied

$$
\boldsymbol{F}_{2}=\boldsymbol{S}_{S} \boldsymbol{F}_{1} \text { and } \boldsymbol{d}_{2}=\boldsymbol{I}_{S} \boldsymbol{d}_{1}
$$

In the simple case of equally sized square stiffness matrices $\boldsymbol{K}_{1}$ and $\boldsymbol{K}_{2}$, the similarity matrix is $\boldsymbol{S}_{S}=\boldsymbol{K}_{2} \boldsymbol{K}_{1}^{-1}$. However, this approach requires static condensation of the structure matrix that is not always practical or possible. Notice that the Sylvester equation does not have the limit of $\boldsymbol{K}$ matrices being of the same size. Here is a formulation of the similarity matrix $S_{S}$ for the general case of $\boldsymbol{K}$ matrices being of different size $\left(\boldsymbol{K}_{1} \leftrightarrow[n \times n]\right.$ and $\left.\boldsymbol{K}_{2} \leftrightarrow[(m+n) \times(m+n)]\right)$. Let us assume

$$
\begin{gathered}
\boldsymbol{K}_{2}=\left[\begin{array}{cc}
\boldsymbol{A}_{2}^{m \times m} & \boldsymbol{C}_{2}^{m \times n} \\
\boldsymbol{C}_{2}^{T} & \boldsymbol{B}_{2}^{n \times n}
\end{array}\right] ; \quad \boldsymbol{d}_{2}=\left\{\begin{array}{c}
\boldsymbol{d}_{2 A}^{m \times 1} \\
\boldsymbol{d}_{2 B}^{n \times 1}
\end{array}\right\} ; \\
\boldsymbol{F}_{2}=\left\{\begin{array}{l}
\boldsymbol{F}_{2 A}^{m \times 1} \\
\boldsymbol{F}_{2 B}^{n \times 1}
\end{array}\right\}
\end{gathered}
$$

and

$$
\boldsymbol{K}_{2}=\boldsymbol{S}_{S} \boldsymbol{K}_{1} \text { with } \boldsymbol{S}_{S}=\left[\begin{array}{c}
\boldsymbol{S}_{A}^{m \times n} \\
\boldsymbol{S}_{B}^{n \times n}
\end{array}\right]
$$

Applying the constraint $\boldsymbol{d}_{2 B}=\boldsymbol{I}_{S} \boldsymbol{d}_{1}=\boldsymbol{d}_{1}$ from Eq. (5) and expanding Eq. (8) one gets

$$
\begin{aligned}
& {\left[\begin{array}{ll}
\boldsymbol{A}_{2} & \boldsymbol{C}_{2} \\
\boldsymbol{C}_{2}^{T} & \boldsymbol{B}_{2}
\end{array}\right]\left\{\begin{array}{c}
\boldsymbol{d}_{2 A} \\
\boldsymbol{d}_{1}
\end{array}\right\}=\left[\begin{array}{c}
\boldsymbol{S}_{A} \\
\boldsymbol{S}_{B}
\end{array}\right]\left[\boldsymbol{K}_{1}\right]\left\{\boldsymbol{d}_{1}\right\}=} \\
& =\left[\begin{array}{l}
\boldsymbol{S}_{A} \\
\boldsymbol{S}_{B}
\end{array}\right]\left[\begin{array}{ll}
\boldsymbol{O} & \boldsymbol{K}_{1}
\end{array}\right]\left\{\begin{array}{c}
\boldsymbol{d}_{2 A} \\
\boldsymbol{d}_{1}
\end{array}\right\}
\end{aligned}
$$

From here it follows

$$
\begin{gathered}
{\left[\begin{array}{ll}
\boldsymbol{A}_{2} & \boldsymbol{C}_{2} \\
\boldsymbol{C}_{2}^{T} & \boldsymbol{B}_{2}
\end{array}\right]=\left[\begin{array}{ll}
\boldsymbol{S}_{A} \boldsymbol{0} & \boldsymbol{S}_{A} \boldsymbol{K}_{1} \\
\boldsymbol{S}_{B} \boldsymbol{0} & \boldsymbol{S}_{B} \boldsymbol{K}_{1}
\end{array}\right] \text { and }} \\
{\left[\begin{array}{l}
\boldsymbol{C}_{2} \\
\boldsymbol{B}_{2}
\end{array}\right]=\left[\begin{array}{l}
\boldsymbol{S}_{A} \\
\boldsymbol{S}_{B}
\end{array}\right]\left[\boldsymbol{K}_{1}\right]}
\end{gathered}
$$

Finally, the two parts of the similarity matrix are 


$$
\begin{aligned}
& \boldsymbol{S}_{S}^{(m+n) \times n}=\left[\begin{array}{l}
\boldsymbol{S}_{A} \\
\boldsymbol{S}_{B}
\end{array}\right] \text { with } \boldsymbol{S}_{A}^{m \times n}=\boldsymbol{C}_{2} \boldsymbol{K}_{1}^{-1} ; \\
& \boldsymbol{S}_{B}^{n \times n}=\boldsymbol{B}_{2} \boldsymbol{K}_{1}^{-1}
\end{aligned}
$$

When stiffness matrices $\boldsymbol{K}_{1}$ and $\boldsymbol{K}_{2}$ are of the same size, $\boldsymbol{A}_{2}=0 ; \boldsymbol{C}_{2}=0$ and $\boldsymbol{B}_{2}=\boldsymbol{K}_{2}$, we recover the simple equation for the similarity matrix $\boldsymbol{S}_{S}=\boldsymbol{K}_{2} \boldsymbol{K}_{1}^{-1}$.

\subsection{Similarity of structures under dynamic loading}

In the case of static loading, the equation of the structure model describes the equilibrium of internal and external forces. The same equilibrium in the case of dynamic loading is described with D'Alembert's system of equations. D'Alembert's equilibrium equation of dynamically loaded structures includes two types of forces: inertial and elastic. We will ignore damping and the relating equation now involves two similarity matrices $\boldsymbol{S}_{S}-$ static similarity matrix and $\boldsymbol{S}_{D}$ - dynamic similarity matrix

$$
\boldsymbol{S}_{D} \boldsymbol{M}_{1} \ddot{\boldsymbol{d}}_{1}(t)+\boldsymbol{S}_{S} \boldsymbol{K}_{1} \boldsymbol{d}_{1}(t)=\boldsymbol{M}_{2} \ddot{\boldsymbol{d}}_{2}(t)+\boldsymbol{K}_{2} \boldsymbol{d}_{2}(t)
$$

where $\boldsymbol{M}$ and $\boldsymbol{K}$ are mass and stiffness matrices of structures ' 1 ' and '2' respectively. From Eqs. (5) and (7) it can be obtained

$$
\boldsymbol{d}_{2}=\left\{\begin{array}{c}
\boldsymbol{d}_{2 A} \\
\boldsymbol{I}_{S} \boldsymbol{d}_{1}
\end{array}\right\} \quad \text { and } \quad \frac{d^{2}}{d t^{2}} \boldsymbol{d}_{2}=\left\{\begin{array}{c}
\ddot{\boldsymbol{d}}_{2 A} \\
\boldsymbol{I}_{S} \ddot{\boldsymbol{d}}_{1}
\end{array}\right\}
$$

Now, we write (without explicitly writing the time variable $t$ )

$$
\left[\begin{array}{l}
\boldsymbol{S}_{D A} \\
\boldsymbol{S}_{D B}
\end{array}\right] \boldsymbol{M}_{1} \ddot{\boldsymbol{d}}_{1}+\left[\begin{array}{c}
\boldsymbol{S}_{S A} \\
\boldsymbol{S}_{S B}
\end{array}\right] \boldsymbol{K}_{1} \boldsymbol{d}_{1}=\left[\begin{array}{cc}
\boldsymbol{M}_{2 A} & \boldsymbol{0} \\
\boldsymbol{0} & \boldsymbol{M}_{2 B}
\end{array}\right]\left\{\begin{array}{l}
\ddot{\boldsymbol{d}}_{2 A} \\
\boldsymbol{I}_{S} \ddot{\boldsymbol{d}}_{1}
\end{array}\right\}+\left[\begin{array}{cc}
\boldsymbol{A}_{2} & \boldsymbol{C}_{2} \\
\boldsymbol{C}_{2}^{T} & \boldsymbol{B}_{2}
\end{array}\right]\left\{\begin{array}{c}
\boldsymbol{d}_{2 A} \\
\boldsymbol{I}_{S} \boldsymbol{d}_{1}
\end{array}\right\}
$$

We separately relate the static and the dynamic part and, after expanding as in Eq. (9), the static part reads

$$
\left[\begin{array}{c}
\boldsymbol{S}_{S A} \\
\boldsymbol{S}_{S B}
\end{array}\right]\left[\begin{array}{ll}
\boldsymbol{0} & \boldsymbol{K}_{1}
\end{array}\right]\left\{\begin{array}{c}
\boldsymbol{d}_{2 A} \\
\boldsymbol{d}_{1}
\end{array}\right\}=\left[\begin{array}{ll}
\boldsymbol{A}_{2} & \boldsymbol{C}_{2} \\
\boldsymbol{C}_{2}^{T} & \boldsymbol{B}_{2}
\end{array}\right]\left\{\begin{array}{c}
\boldsymbol{d}_{2 A} \\
\boldsymbol{I}_{S} \boldsymbol{d}_{1}
\end{array}\right\}
$$

From Eq. (15) we obtain equation similar to Eq. (10)

$$
\begin{aligned}
{\left[\begin{array}{l}
\boldsymbol{C}_{2} \boldsymbol{I}_{S} \\
\boldsymbol{B}_{2} \boldsymbol{I}_{S}
\end{array}\right] } & =\left[\begin{array}{l}
\boldsymbol{S}_{S A} \\
\boldsymbol{S}_{S B}
\end{array}\right]\left[\boldsymbol{K}_{1}\right] \text { and } \boldsymbol{S}_{S A}=\boldsymbol{C}_{2} \boldsymbol{I}_{S} \boldsymbol{K}_{1}^{-1} ; \\
\boldsymbol{S}_{S B} & =\boldsymbol{B}_{2} \boldsymbol{I}_{S} \boldsymbol{K}_{1}^{-1}
\end{aligned}
$$

The dynamic part reads

$$
\left[\begin{array}{l}
\boldsymbol{S}_{D A} \\
\boldsymbol{S}_{D B}
\end{array}\right]\left[\begin{array}{ll}
\boldsymbol{0} & \boldsymbol{M}_{1}
\end{array}\right]\left\{\begin{array}{c}
\ddot{\boldsymbol{d}}_{2 A} \\
\ddot{\boldsymbol{d}}_{1}
\end{array}\right\}=\left[\begin{array}{cc}
\boldsymbol{M}_{2 A} & \boldsymbol{0} \\
\boldsymbol{0} & \boldsymbol{M}_{2 B}
\end{array}\right]\left\{\begin{array}{l}
\ddot{\boldsymbol{d}}_{2 A} \\
\boldsymbol{I}_{S} \ddot{\boldsymbol{d}}_{1}
\end{array}\right\}
$$

From Eq. (17) we obtain

$$
\begin{gathered}
{\left[\begin{array}{c}
\boldsymbol{0} \\
\boldsymbol{M}_{2 B} \boldsymbol{I}_{S}
\end{array}\right]=\left[\begin{array}{l}
\boldsymbol{S}_{D A} \\
\boldsymbol{S}_{D B}
\end{array}\right]\left[\boldsymbol{M}_{1}\right] \text { and } \boldsymbol{S}_{D A}=\boldsymbol{0} ;} \\
\boldsymbol{S}_{D B}=\boldsymbol{M}_{2 B} \boldsymbol{I}_{S} \boldsymbol{M}_{1}^{-1}
\end{gathered}
$$

In the case of equally sized matrices, $\boldsymbol{M}_{2 A}$ and $\boldsymbol{S}_{D A}$ disappear from Eq. (17) and we have

$$
\left[\boldsymbol{S}_{D B}\right]\left[\boldsymbol{M}_{1}\right]\left\{\ddot{\boldsymbol{d}}_{1}\right\}=\left[\boldsymbol{M}_{2 B}\right]\left\{\boldsymbol{I}_{S} \ddot{\boldsymbol{d}}_{1}\right\}
$$

and the dynamic scaling matrix is $\boldsymbol{S}_{D}=\boldsymbol{M}_{2} \boldsymbol{I}_{S} \boldsymbol{M}_{1}^{-1}$ (index ' $B$ ' is no longer required since there is no ' $A$ ' part for matrices of the same size). Of course, the static scaling matrix simplifies to $\boldsymbol{S}_{S}=\boldsymbol{K}_{2} \boldsymbol{I}_{S} \boldsymbol{K}_{1}^{-1}$.

In order to verify the scaling matrices formulation we compare the dynamic matrices of two structures by rewriting Eq. (12) in the form of the eigenvalue problem

$-\omega_{1}^{2} \boldsymbol{S}_{D} \boldsymbol{M}_{1} \boldsymbol{I}_{S} \boldsymbol{d}_{1}(t)+\boldsymbol{S}_{S} \boldsymbol{K}_{1} \boldsymbol{I}_{S} \boldsymbol{d}_{1}(t)=\mathbf{0}$

and

$-\omega_{2}^{2} \boldsymbol{M}_{2} \boldsymbol{d}_{2}(t)+\boldsymbol{K}_{2} \boldsymbol{d}_{2}(t)=\boldsymbol{0}$

with the dynamic matrix $\boldsymbol{D}_{2}=\left(\boldsymbol{M}_{2}\right) \cdot\left(\boldsymbol{K}_{2}\right)^{-1}$. It is easy to prove that the two dynamic matrices are equivalent by substituting the above expressions for $\boldsymbol{S}_{D}$ and $\boldsymbol{S}_{S}$ into Eq. (20). Consequently, eigenvalues and eigenvectors have to be the same, i.e. $\omega_{1}=\omega_{2}$.

The scaling matrices $\boldsymbol{S}_{S}$ and $\boldsymbol{S}_{D}$ can be determined and are well conditioned if the structural mass and stiffness matrices are well conditioned. In addition, mass matrices $\boldsymbol{M}_{1}$ and $\boldsymbol{M}_{2}$ are in many cases lumped, i.e. diagonal in which case $\boldsymbol{S}_{S}$ and $\boldsymbol{S}_{D}$ are easily calculated. Structures related with $\boldsymbol{S}_{S}$ and $\boldsymbol{S}_{D}$ scaling matrices have the same eigenvalues and eigenvectors, i.e. resonant frequencies are the same and the response to dynamic loading is the same. The relationship between dynamic matrices $\boldsymbol{D}_{1}$ and $\boldsymbol{D}_{2}$ is

$\boldsymbol{P}=\boldsymbol{D}_{2} \boldsymbol{D}_{1}^{-1} \quad$ with $\quad \boldsymbol{P}=\boldsymbol{S}_{D} \boldsymbol{M}_{1} \boldsymbol{K}_{1}^{-1} \boldsymbol{S}_{S}^{-1} \boldsymbol{K}_{1} \boldsymbol{M}_{1}^{-1}$

This is only one possibility of expressing scaling matrix $\boldsymbol{P}$, one could write

$$
\boldsymbol{P}=\boldsymbol{D}_{2} \boldsymbol{D}_{1}^{-1}=\boldsymbol{S}_{D} \boldsymbol{D}_{1} \boldsymbol{S}_{S}^{-1} \boldsymbol{D}_{1}^{-1}
$$

From here follows

$\boldsymbol{D}_{2}=\boldsymbol{S}_{D} \boldsymbol{D}_{1} \boldsymbol{S}_{S}^{-1}$

Mathematical justification for the above procedure can be found in the Smith normal form $\boldsymbol{S}(\boldsymbol{x})=\boldsymbol{X} \cdot \boldsymbol{I}_{n}-\boldsymbol{A}$ where $\boldsymbol{X}$ is a polynomial matrix (just like the characteristic matrix for the eigenproblem). Two matrices are similar if and only if their Smith normal forms are equal. In our problem, matrix $\boldsymbol{A}$ is the dynamic matrix $\boldsymbol{D}$ and we see that if $\boldsymbol{D}_{1}$ and $\boldsymbol{D}_{2}$ are similar, eigenvalues and eigenvectors are the same (and vice versa).

One consequence of this property of dynamic matrices is that the direct scaling of eigenvalues and 
eigenvectors is not so straightforward without first determining the dynamic matrices $\boldsymbol{D}_{1}$ and $\boldsymbol{D}_{2}$. Direct scaling is possible, as it can be seen from the aposteriori analysis

$$
\boldsymbol{L}_{2} \boldsymbol{L}_{1}^{-1}=\boldsymbol{S}_{L}, \quad \boldsymbol{Q}_{2} \boldsymbol{Q}_{1}^{-1}=\boldsymbol{S}_{Q}
$$

Here, $\boldsymbol{S}_{L}$ and $\boldsymbol{S}_{Q}$ are eigenvalue and eigenvector scaling matrices, $\boldsymbol{L}$ is a diagonal matrix with eigenvalues on the diagonal and $\boldsymbol{Q}$ is the eigenvector matrix. Applying the eigenvalue decomposition theorem one could write

$$
\begin{aligned}
\boldsymbol{D}_{1}= & \boldsymbol{Q}_{1} \boldsymbol{L}_{1} \boldsymbol{Q}_{1}^{-1}, \quad \boldsymbol{D}_{2}=\boldsymbol{Q}_{2} \boldsymbol{L}_{2} \boldsymbol{Q}_{2}^{-1} \\
\boldsymbol{D}_{2}= & \boldsymbol{S}_{Q} \boldsymbol{Q}_{1} \boldsymbol{L}_{2} \boldsymbol{Q}_{1}^{-1} \boldsymbol{S}_{Q}^{-1} \text { and } \\
& \boldsymbol{D}_{2} \boldsymbol{S}_{Q}=\boldsymbol{S}_{Q} \boldsymbol{Q}_{1} \boldsymbol{L}_{2} \boldsymbol{Q}_{1}^{-1}=\boldsymbol{S}_{Q} \boldsymbol{D}_{12}
\end{aligned}
$$

from the eigenvalue problem

$$
\boldsymbol{D}_{2} \boldsymbol{S}_{Q}-\boldsymbol{S}_{Q} \boldsymbol{D}_{12}=\mathbf{0}
$$

$\boldsymbol{S}_{Q}$ could be determined following Eq. (2), unfortunately only up to a diagonal matrix [10]. Of course, one could say that this is the consequence of the non-linear character of the eigenvalue problem and that it could not be fitted into the linear relationship. In this paper, scaling of eigenvalues and eigenvectors has been performed only after dynamic matrices $\boldsymbol{D}_{1}$ and $\boldsymbol{D}_{2}$ have been solved for eigenvalues and eigenvectors.

\subsection{Similarity of structures through equal parameters}

In the case of load/displacement similarity/scaling, we were making load and displacement vectors proportional through a specially designed matrix $\boldsymbol{S}$. Loading and displacement could exchange their places if the stiffness matrix is replaced with the flexibility matrix.

As we have seen above, relating stiffness matrices is much more demanding, so we have reverted to an optimization procedure: Levenberg-Marquardt is used to determine stiffness matrix parameters that would make the displacements equal/related as requested.

The procedure is as follows

$$
\boldsymbol{e}(\boldsymbol{k})=\sum_{i=0}^{n}\left(\delta_{i}^{m}-\delta(\boldsymbol{k})_{i}\right)^{2}
$$

where $\boldsymbol{k}$ is the vector of stiffness parameters, $\boldsymbol{\delta}^{m}$ is the vector of required displacements and $\boldsymbol{\delta}(\boldsymbol{k})$ is the calculated displacement vector that is a function of stiffness parameters. The optimization condition is

$$
\frac{d}{d \boldsymbol{k}} \boldsymbol{e}(\boldsymbol{k})=\boldsymbol{0}=\sum_{i=0}^{n}\left(\delta_{i}^{m}-\delta(\boldsymbol{k})_{i}\right) \boldsymbol{X}_{i}
$$

where $\boldsymbol{X}_{i}=\frac{d}{d \boldsymbol{k}} \delta(\boldsymbol{k})_{i}$ is the vector of sensitivity parameters.

Taylor expansion gives
$\delta(\boldsymbol{k}+\Delta \boldsymbol{k})_{i}=\delta(\boldsymbol{k})_{i}+\frac{d \delta_{i}}{d \boldsymbol{k}} \Delta \boldsymbol{k}$

so Eq. (29) becomes

$\sum_{i=0}^{n}\left(\delta_{i}^{m}-\delta(\boldsymbol{k})_{i}-\frac{d \delta_{i}}{d \boldsymbol{k}} \Delta \boldsymbol{k}\right) \boldsymbol{X}_{i}=0$

and finally, the increment of the vector of stiffness parameters is

$\Delta \boldsymbol{k}=\frac{\sum_{i=0}^{n}\left(\delta_{i}^{m}-\delta(\boldsymbol{k})_{i}\right)}{\sum_{i=0}^{n} \boldsymbol{X}_{i}^{2}}$

Update of the vector of stiffness parameters is as given by Eq. (4). Since this is an optimization procedure, the resulting stiffness matrix only approximately satisfies the required condition, i.e. displacements are only approximately equal.

\section{Examples \\ 4.1 Example 1: Similarity between truss and beam}

All the examples are calculated in Wolfram Mathematica [11] and PTC MathCad [12].

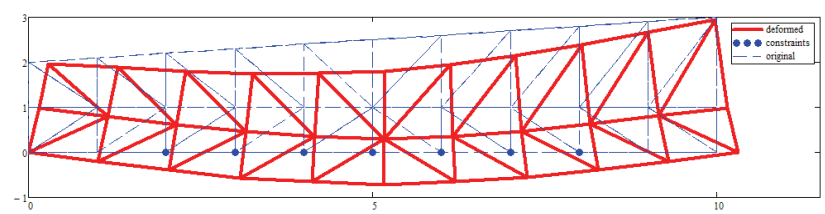

Figure 1 Truss: nodes for comparison

Two completely different structures, a truss and a beam, are scaled in parameter and in measurement space. Structural properties are (see Fig. 1):

Truss: $E A=1000,0 \mathrm{kN}, L=10,0 \mathrm{~m}, h=2,0 \mathrm{~m}$, discretized using finite element method with 33 nodes, 72 bars; total number of degrees of freedom (size of the stiffness matrix) $m+n=66$.

Beam: $\quad E I=1000,0 \mathrm{kNm}^{2}, L=1,0 \mathrm{~m}$, discretized using finite difference method with 21 node, 20 beams; total number of degrees of freedom (size of the stiffness matrix) $n=21$.

Truss and beam not only have different number of degrees of freedom but also are different regarding symmetry. We would like to relate the seven displacements marked in Fig. 1 and Fig. 2 so that they are equal (in practice we would not set them to be equal, but in that case the results would not be so intuitive. For example, beam displacements could be 10 times smaller than truss displacements as that relation reflects their length ratio).

Scaling matrix $\boldsymbol{S}_{S}$ has to be determined so that displacements in marked nodes are related (the same in this case, $\boldsymbol{I}_{S}=\boldsymbol{I}$ ). The beam in Fig. 2 is loaded in an unusual way to make the comparison more interesting. Index ' 1 ' stands for beam and index ' 2 ' for truss. Truss 
node numbers for relating the displacements are: (7 10 $\left.\begin{array}{lllll}13 & 16 & 19 & 22 & 25\end{array}\right)$. Node numbers are important because the stiffness matrix has to be re-assembled in partitioned form as in Eq. (7). Care is needed regarding the corresponding equation numbers since they change during the matrix partitioning.

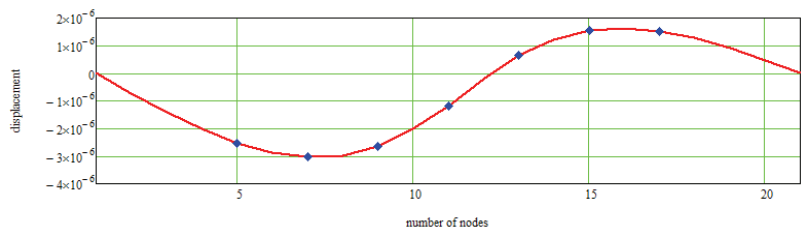

Figure 2 Beam: nodes for comparison

The corresponding beam node numbers are: $\left(\begin{array}{lll}5 & 7 & 9\end{array}\right.$ $11 \quad 13 \quad 15$ 17); the beam stiffness matrix has to be partitioned, too. The consequence of partitioning is that matrices are no longer banded but they remain symmetric (due to simultaneous rearranging of both rows and columns).

\subsubsection{Similarity matrix for displacement scaling}

In this example, we are scaling truss forces so that displacements in the beam and in the truss have the same value at selected nodes (or any desired value set by the matrix $\boldsymbol{I}_{\boldsymbol{S}}$ ). The scaling matrix is given with Eq. (11).

Comparison of the results is illustrated in Fig. 3.

This example demonstrates how we can scale the loading of two completely different structures (i.e. a large structure and a small model) and obtain the same displacements. The scaling matrix is independent of the loading and has to be determined only once for every structure pair. Also, matrix $\boldsymbol{S}_{x}$ is dense, so all selected points on two structures are related.

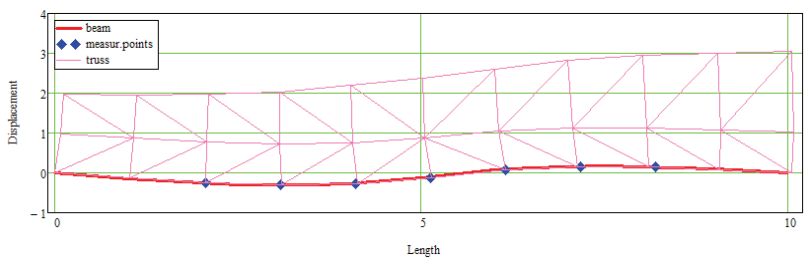

Figure 3 Comparison of displacements of truss and beam with scaled loading

\subsubsection{Similarity matrix for load scaling}

In this example, we are scaling truss displacements so that they are equal to beam displacements under the same loading. Two single displacement vectors can be related in an arbitrary way. One possibility is to use a generalized inverse matrix in the form $\boldsymbol{S}_{y}=\boldsymbol{y}_{2}^{\mathrm{T}}\left(\boldsymbol{y}_{2} \boldsymbol{y}_{2}^{\mathrm{T}}\right)^{-1} \boldsymbol{y}_{1}$ that corresponds to the minimum length solution. However, scaling results are poor under general loading (for single loading case $\boldsymbol{S}_{y}$ degenerates into a scalar). Under the assumption of independence of displacements of the truss and the beam, the scaling matrix $\boldsymbol{S}_{y}$ is diagonal with elements

$$
\left(\boldsymbol{S}_{y}\right)_{i, i}=\frac{\left(y_{1}\right)_{i}}{\left(y_{2}\right)_{i}}
$$

in which case displacements are exactly scaled.

\subsubsection{Scaling of dynamic properties}

We would like to compare eigenfrequencies and modal shapes (eigenvalues) of a model and a structure, so they have to be scaled. In our example, the structure is the truss and the model is the beam from above, and both have lumped mass matrices. Truss and beam eigenmodes are presented in Fig. 4

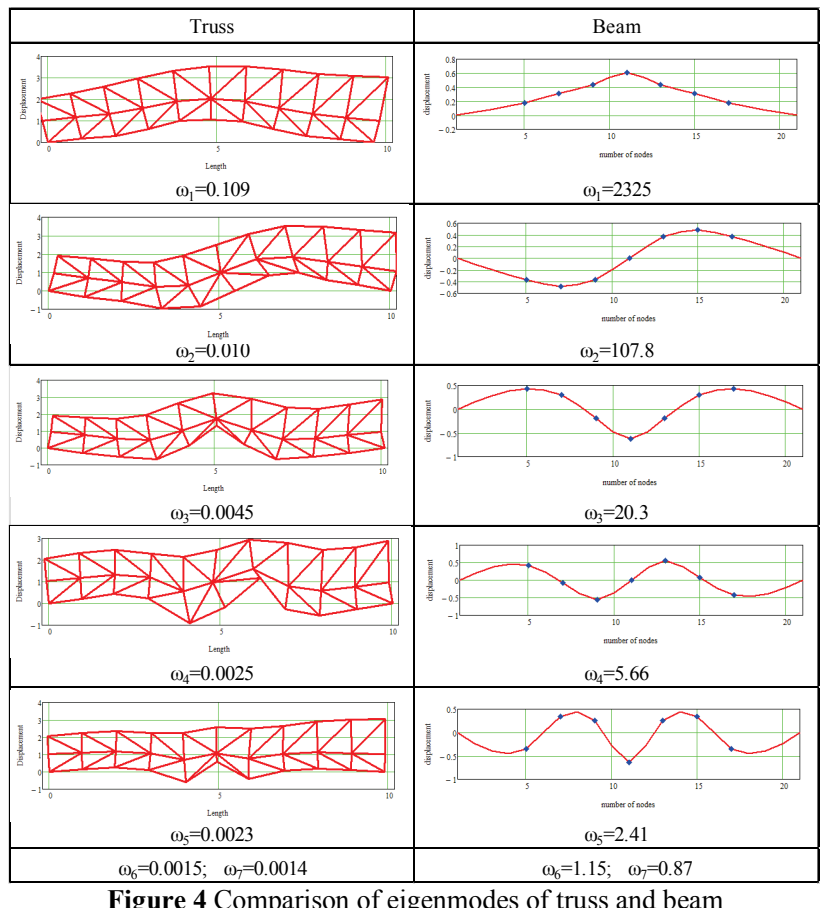

Eigenmodes in Fig. 4 cannot be scaled directly; instead, mass and stiffness matrices have to be scaled to produce the same eigenvalues. Matrix $\boldsymbol{I}_{S}$ from Eq. (13) is made equal to $\boldsymbol{S}_{y}$ from example 4.1.2, producing scaling matrices $\boldsymbol{S}_{s}=\boldsymbol{K}_{1} \boldsymbol{I}_{S} \boldsymbol{K}_{2}^{-1}$ and $\boldsymbol{S}_{d}=\boldsymbol{M}_{1}\left(\boldsymbol{I}_{S}^{2}\right)^{-1} \boldsymbol{M}_{2}^{-1}$. The scaled truss dynamic matrix $\boldsymbol{D}_{s}=\left(\boldsymbol{S}_{d} \boldsymbol{M}_{2} \boldsymbol{I}_{S}^{2}\right)\left(\boldsymbol{S}_{s} \boldsymbol{K}_{2} \boldsymbol{I}_{S}^{-1}\right)^{-1}$ has the same eigenvalues and eigenvectors as the beam dynamic matrix $\boldsymbol{D}_{1}=\boldsymbol{M}_{1} \boldsymbol{K}_{\text {con }}^{-1}$. Now, relating the appropriate dynamic matrices we can scale eigenvalues using the eigenvalue decomposition theorem: from ' $r$ ' measured eigenvalues $\Omega$ and eigenvectors $\boldsymbol{\Phi}$, dynamic matrices are formed

$\boldsymbol{D}=\sum_{r} \Omega_{r} \boldsymbol{\Phi}_{r}\left(\Phi_{r}^{-g}\right)^{\mathrm{T}}$

and eigenmodes are related.

\subsection{Example 2: Similarity for replacing beam part with equivalent truss}

In this example, we are removing a part of the beam that we would like to replace with a truss so that the new structure has the same stiffness as the beam. The beam properties are: $L=10,0 \mathrm{~m}$ and $E I=1000,0 \mathrm{kNm}^{2}$, the 
discretization method is finite differences. The middle 0,5 meters of the beam are to be removed and replaced with the truss. The truss length is $L=2,0 \mathrm{~m}$, stiffness is to be determined; the discretization method is finite elements, geometry is depicted in Fig. 5.

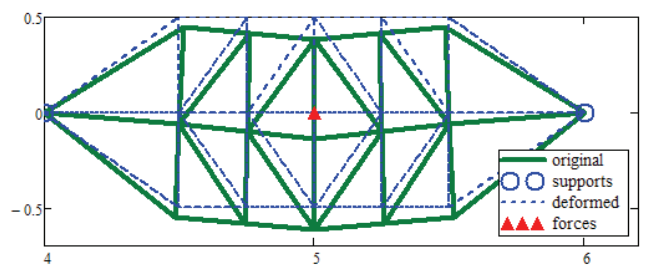

Figure 5 Replacement truss with added supports and loading in the middle

In Fig. 5, supports have been added to the truss so that it can be loaded and displacements can be calculated.

The main difficulty of this example is that the beam becomes singular (unstable) when a part is removed. In addition, the truss to be inserted must not have supports, so it is singular, too. However, when we combine the beam and the truss, the resulting structure is not singular. One can naively combine the structures and try to adjust the truss stiffness to obtain equivalent displacements. The result is presented in Fig. 6.

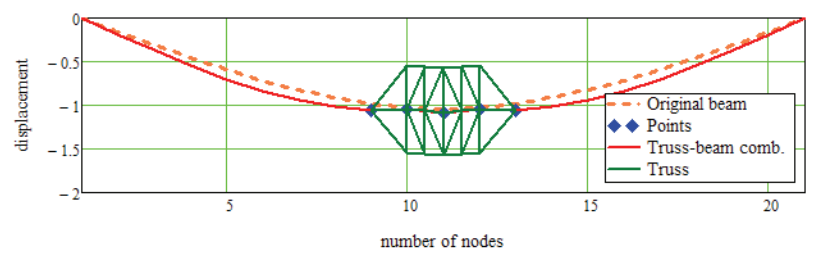

Figure 6 Beam-truss combination without similarity matrix

In Fig. 6, it can be seen that we could adjust the displacements in only two points, and the result is not satisfactory. Moreover, for different loading one has to adjust the stiffness from the beginning.

Determination of the similarity matrix as given in Eq. (11) and its application on the truss stiffness matrix resolves the problem. It is important to correctly recognize matrices for the similarity condition that enters Eq. (11): singular truss (truss without supports) + singular beam (beam without the middle part) = combined beamtruss (not singular).

Since matrices that enter the equation are singular, all inverse matrices are to be replaced with generalized matrix inverse (as in [13]). It is also helpful if rigid body modes are removed from stiffness matrices [14], which is not an imperative with a good algorithm for generalized matrix inverse (e.g. with application of singular value decomposition if necessary, see [15]).

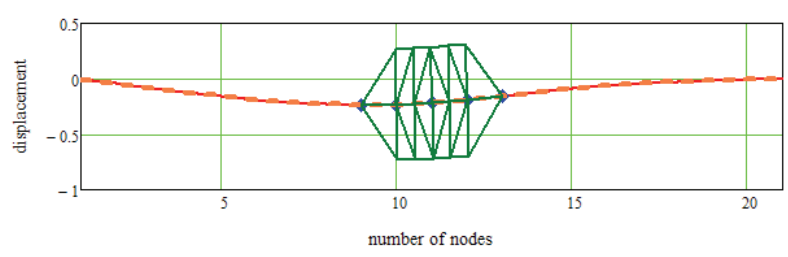

Figure 7 Beam-truss combination with application of similarity matrix
In Fig. 7, we see that the new combined beam-truss structure is completely compatible with the beam regarding displacements in the chosen nodes and it is so for any (compatible) loading.

\subsection{Example 3: Stiffness parameter calculation for truss to match beam}

In the above examples, we have adjusted the structure stiffness by multiplying it with the similarity matrix $S$ obtaining thus the desired stiffness. That is enough for numerical calculations and comparison of forces and displacements. However, it is of no use if we would like to produce a structure of desired stiffness, i.e. if we know only the resulting values in the stiffness matrix. If we need dimensions of structure members, they have to be determined using a separate procedure. Here, we have chosen Levenberg-Marquardt procedure, and Eq. (4) and Eq. (32) have to be employed.

We would like to determine the stiffness of truss members so that displacements at given points of the truss equal those at given points of the beam. In our example there are two parameters: stiffness of the upper and stiffness of the lower truss diagonals, as shown in Fig. 9. The number of parameters is an important factor; the size of the Jacobian in Eq. (4) depends on the number of stiffness parameters we choose for the truss. With the increase in Jacobian size we increase the sensitivity of the Levenberg-Marquardt numerical procedure.

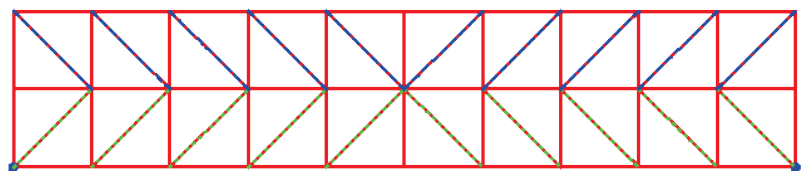

Figure 8 Truss with upper and lower diagonals as stiffness parameters

The truss length is $L=10,0 \mathrm{~m}$ and the starting stiffness for all members is $E A=1000,0 \mathrm{kN}$. The displacements of the truss should correspond to the beam.

The beam length is $L=10,0 \mathrm{~m}$ and its bending stiffness is $E I=1000,0 \mathrm{kNm}^{2}$. Note: we do not put stiffness into relation since it is of different type, bending vs. extension stiffness. We relate displacements, requiring them to be equal.

The result of the optimization procedure is $E A_{l}=$ $243,65 \mathrm{kN}$ and $E A_{u}=473,25 \mathrm{kN}$ for the stiffness of lower and upper diagonals, and comparison of the results is given in Tab. 1.

Table 1 Comparison of displacements between beam and truss

\begin{tabular}{|c|c|c|c|c|c|c|c|}
\hline Point & 1 & 2 & 3 & 4 & 5 & 6 & 7 \\
\hline Beam & 0,0346 & 0,0482 & 0,0573 & 0,0604 & 0,0573 & 0,0482 & 0,0346 \\
\hline Truss & 0,0338 & 0,0488 & 0,0562 & 0,0620 & 0,0562 & 0,0488 & 0,0338 \\
\hline $\begin{array}{c}\text { Error } \\
\% \text { WM }\end{array}$ & $-2,34$ & 1,27 & $-1,76$ & 2,58 & $-1,76$ & 1,27 & $-2,34$ \\
\hline $\begin{array}{c}\text { Error } \\
\% \text { PTC }\end{array}$ & 0,005 & 4,052 & 0,517 & 5,140 & 0,517 & 4,052 & 0,005 \\
\hline
\end{tabular}

Sensitivity of the procedure is best seen if we compare results from Mathematica10 and Mathcad 14. Although the procedures are equal, Mathcad gives $E A_{l}=$ $351,47 \mathrm{kN}$ and $E A_{u}=275,33 \mathrm{kN}$ for the stiffness of lower and upper diagonals. The maximum error for the first case is around 2,6\% compared to 5,1\% for the second. The reason for this behaviour is clear if we construct and plot 
the error as a function of diagonal member stiffness $E R\left(E A_{l}, E A_{w}\right)$, as presented in Fig. 9 (semi-log plot).

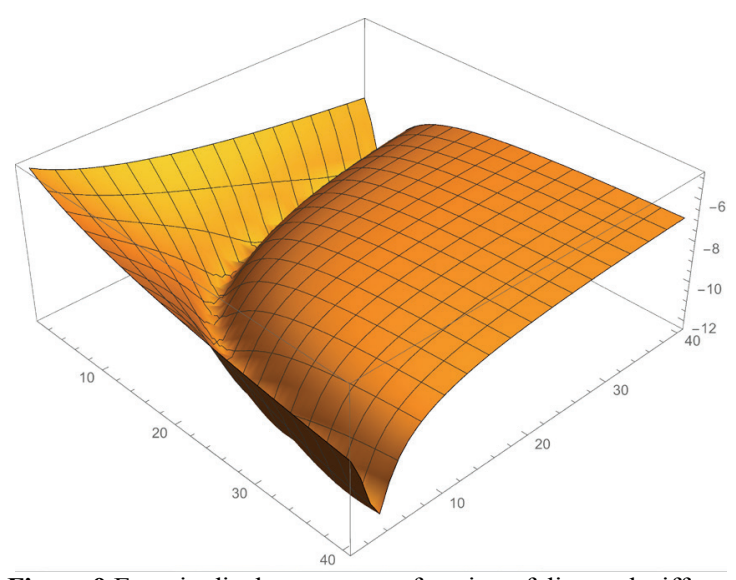

Figure 9 Error in displacement as a function of diagonal stiffness

Fig. 9 shows that there is a broad area of convergence and that there are many pairs of diagonal stiffness that minimize the error with only slight differences in error magnitude. Consequently, there are many solutions to the problem. Some different loading has a different area of convergence and optimal solution for several loading cases is within an intersection of corresponding convergence areas.

\section{Discussion and conclusion}

This paper presents a novel method for relating force and displacement vectors of various, completely different structures. The solution is based on the notion of matrix similarity and linear transformations, which requires that structures are discretized prior to scaling and that displacements are in the elastic regime. This relation between vectors resembles scaling in physical space and the term "scaling" is used in that sense, since it allows us to establish relation between two structures of different size without restriction of dimensional analysis. The relation between vectors can be determined in loading space or in displacement space (i.e. structure forces or displacements are put into relation) separately for the static and the dynamic model. The relation between vectors is obtained in the form of a matrix called "similarity matrix". Scaling of the static structure model is based on the matrix equivalence principle between two stiffness matrices. Scaling of the dynamic structure model is based on the Smith normal form used to put into relation dynamic matrices of two structures. The result is a similarity matrix relating eigenvectors of two structures.

Extension of the method is towards relating stiffness matrices, i.e. scaling in the operator space (structure stiffness or flexibility matrices are put into relation). It should be based on the Sylvester matrix equation. However, that approach is not practical for engineering use and is replaced with the Levenberg-Marquardt method for obtaining only approximately equivalent stiffness matrices. This method does not belong to linear transformations any more but is an optimization method. As a consequence, the solution is only approximate and related to only one load - displacement pair. Optimal solution for an interval of load - displacement pairs is an intersection from the set of solutions. In order to keep the optimization error within acceptable bounds, the load interval should not be large; exact values depend on the structure.

Numerical examples illustrate the proposed novel approach, its possible usefulness and its limitations.

\section{Acknowledgment}

This paper has been partially funded through Croatian Science Foundation project 9068: Multi-scale concrete model with parameter identification, which is fully appreciated.

\section{References}

[1] Aster, R. C.; Borchers, B.; Thurber, C. H. Parameter Estimation and Inverse Problems, Academic Press, 2013.

[2] Ghosh, A. Scaling Laws, in Mechanics over Micro and Nano Scales, Editor S. Chakraborty, Springer, 2011. DOI: 10.1007/978-1-4419-9601-5_2

[3] Ramu, M.; Raja, V. P.; Thyla, P. R. Establishment of Structural Similitude for Elastic Models and Validation of Scaling Laws. // KSCE Journal of Civil Engineering. 17, 1(2013), pp. 139-144. DOI: 10.1007/s12205-013-1216-x

[4] Henriksen, R. N. Scale Invariance, Self-Similarity of the Physical World, VileyVCH, 2015. DOI: 10.1002/9783527687343

[5] Kožar, I. Relating Structure and Model, in Computational Methods for Solids and Fluids, Editor A. Ibrahimbegovic, Springer, 2016. DOI: 10.1007/978-3-319-27996-1_7

[6] Schatzman, M. Numerical Analysis, Oxford, 2002

[7] Hu, Q.; Cheng, D. The polynomial solution to the Sylvester matrix equation. // Applied Mathematics Letters, 19, (2006), pp. 859-864. DOI: 10.1016/j.aml.2005.09.005

[8] Ibrahimbegovic, A.; Mamouri S. On Rigid Components and Joint Constraints in Nonlinear Dynamics of Flexible Multibody Systems Employing 3D Geometrically Exact Beam Model. // Computer Methods in Applied Mechanics and Engineering. 188, (2000), pp. 805-831. DOI: 10.1016/S0045-7825(99)00363-1

[9] Liu, G. R.; Han, X. Computational Inverse Techniques in Nondestructive Evaluation. CRC Press, 2003. DOI: 10.1201/9780203494486

[10] Cullen, C. G. Matrices and Linear Transformations, Dover, 1990

[11] Wolfram Language and System, Documentation Center, 2015, http://reference.wolfram.com/language/.

[12] PTC Mathcad, Mathcad 14 Electronic Documentation: Mathcad User's Guide, 2007

[13] Gajić, Z.; Tahir, M.; Qureshi, J. Lypunov Matrix Equation in System Stability and Control, Dover Publications, Inc., 2008

[14] Kožar, I.; Torić Malić, N. Spectral method in realistic modeling of bridges under moving vehicles. // Engineering Structures. $50, \quad(2013), \quad$ pp. 149-157. DOI: 10.1016/j.engstruct.2012.10.024

[15] Menke, W. Geophysical Data Analysis: Discrete Inverse Theory, Academic Press Elsevier, 2012

\section{Authors' addresses}

Ivica Kožar, full Prof.

Tea Rukavina, assistant

Neira Torić-Malić, senior assistant

Faculty of Civil Engineering, University of Rijeka, Radmile Matejčić 3, 51000 Rijeka

051265 993, e-mail: ivica.kozar@gradri.uniri.hr

051265 858, e-mail: tea.rukavina@gradri.uniri.hr 051265 994, e-mail: neira.toric@gradri.uniri.hr 